\title{
Emerging chemistry technologies for a better world
}

Chemistry plays a key role in tackling today's challenges and ensuring a sustainable future. Since 2019, IUPAC has been identifying technologies with the potential to advance our society and improve our quality of life.

\section{Fernando Gomollón-Bel and Javier García-Martínez}

O ur society faces unprecedented challenges. The ongoing COVID-19 pandemic is perhaps the one at the forefront of our minds, but there are many other critical global issues that also require urgent action, such as the climate crisis, the growing instability and cost of energy, and the scarcity of natural minerals and resources. In an attempt to tackle these problems, international bodies have launched bold initiatives such as the Paris Agreement and the United Nations Sustainable Development Goals (SDGs), both of which set ambitious targets to improve our world. The role of chemistry is central to finding and implementing innovative solutions that enable a more sustainable future. Therefore, the
International Union of Pure and Applied Chemistry (IUPAC) is working to highlight the importance of the chemical sciences through different outreach projects, among them an initiative to identify emerging technologies in chemistry.

Raising public awareness in chemistry is one of the goals of IUPAC, an organization that, despite some misconceptions, tackles much more than deciding the names of the elements and ruling on chemical nomenclature. Its divisions and committees also review, update and publish critically important chemical data from atomic weights to thermodynamic constants, and fund chemistry-related outreach projects. For example, in 2019, IUPAC launched the Global Women's Breakfast (https://iupac.org/gwb/) to promote chemistry and celebrate diversity, providing a platform for professional growth and discussion; it has continued as a successful annual event ever since.

During its centenary celebrations in 2019, IUPAC spearheaded the organization of the International Year of the Periodic Table and also launched its Top Ten Emerging Technologies in Chemistry project (https://iupac.org/what-we-do/ top-ten/). Following on from similar successful initiatives by institutions such as the Massachusetts Institute of Technology and the World Economic Forum, each year IUPAC highlights ten emerging chemistry technologies that are judged to have the highest potential to improve our quality
IUPAC Top Ten Emerging Technologies in Chemistry 2021

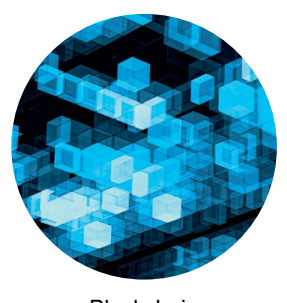

Blockchain

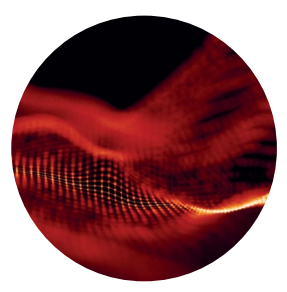

Sonochemical coating

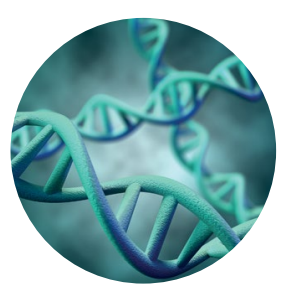

Semisynthetic life

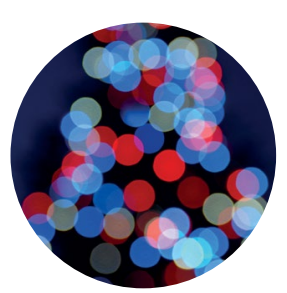

Chemoluminescence

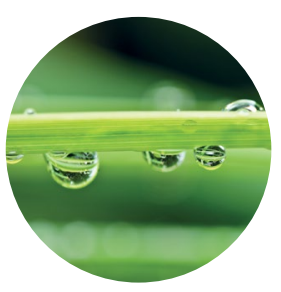

Superwettability

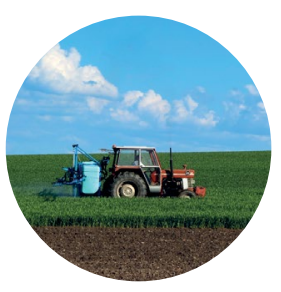

Sustainable ammonia

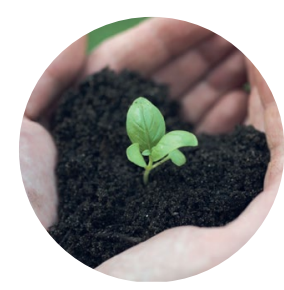

Artificial humus

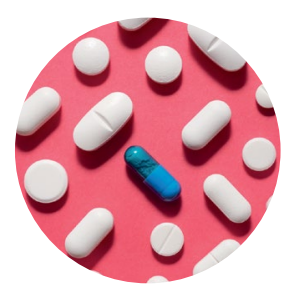

Targeted protein degradation

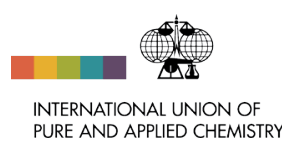
PURE AND APPLIED CHEMISTRY

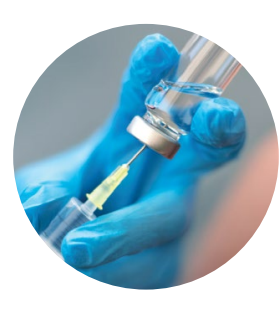

RNA synthesis

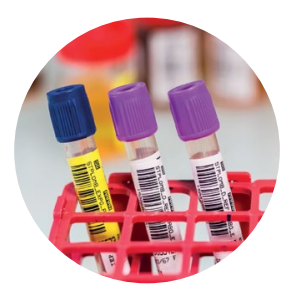

Single-cell metabolomics

Fig. 1 | IUPAC Top Ten Technologies selection for 2021. The newest IUPAC selection for its Top Ten Emerging Technologies in Chemistry initiative, picked for their potential to help achieve a circular economy and a climate-neutral future. Credit: top left and top right, IUPAC; Blockchain, Olga Salt / Alamy Stock Photo; Semisynthetic life, Supparsorn Wantarnagon / Alamy Stock Photo; Superwettability, Peerasith Chaisanit / Alamy Stock Photo; Artificial humus, Claudio Rampinini / Alamy Stock Photo; RNA synthesis, Simon Lehmann / Alamy Stock Photo; Sonochemical coating, Dmytro Razinkov / Alamy Stock Photo; Chemoluminescence, Andrew Paterson / Alamy Stock Photo; Sustainable ammonia, incamerastock / Alamy Stock Photo; Targeted protein degradation, Pedro Antonio Salaverría Calahorra / Alamy Stock Photo; Single-cell metabolomics, Phanie / Alamy Stock Photo 
of life, contribute to our economy and advance society towards a more sustainable future. The chosen technologies are not necessarily the most exciting discoveries of the year, nor well-established industrial solutions. They hover between different stages of development; whereas some are recent experimental endeavours, such as the sustainable production of ammonia, others are commercial, well-established industrial realities, such as RNA vaccines. Any person or institution can suggest ideas through the IUPAC website. After the closing of the submission period, an international panel of experts reviews the pool of submissions and selects those that will appear on the final list. The selection and description of the technologies is shared in an open-access article published ${ }^{1}$ in Chemistry International.

One of society's most difficult challenges is to decouple economic growth from environmental impact and many of the emerging technologies are selected by IUPAC with this in mind ${ }^{2}$. Both in 2019 and 2020, the list included technologies to either manufacture more sustainable plastics or recycle them more easily. Other choices focus on the energy crisis, providing better solutions for clean energy generation and efficient energy storage, through technologies that not only outperform lithium-ion batteries but also are not reliant on scarce materials. The sustainable production of ammonia, one of the 2021 picks (Fig. 1), will have to replace our current solution to fix nitrogen - the Haber-Bosch process - which simultaneously feeds our world while poisoning it with huge amounts of carbon dioxide.

As mentioned at the outset of this Comment, climate and energy are two of the most pressing global challenges and chemists need to help find solutions. As documented by the Intergovernmental Panel on Climate Change (IPCC), the levels of carbon dioxide in our atmosphere are dangerously high. Moreover, energy has reached record-breaking prices during the past few months, mostly due in part to the lack of reliable sustainable solutions and efficient storage ${ }^{3}$. Some of the answers will certainly come from emerging technologies, perhaps including functionalized zeolites and metal-organic frameworks for carbon capture. Other key advances such as photocatalytic water splitting and the manufacture of clean hydrogen will certainly contribute to the generation of 'green' electrons, providing valuable alternatives to traditional renewables. Although these two technologies were absent from the first Top Ten selections, they exemplify ideas for future suggestions and could appear on subsequent lists - especially after the strong push for green hydrogen in the European Union and the United Kingdom, with programmes such as the European Green Deal and the recent COVID-19 Recovery Funds.

The Top Ten lists also venture into the frontiers where the boundary between chemistry and biology blurs, featuring technologies with promising applications in medicine and pharmacology. Among these, mechanochemistry, microbiome studies and targeted protein degradation (TPD) stand out. The first of these enables new transformations without solvents, making chemistry greener and giving access to novel reactivities and selectivities, something quite attractive for the pharmaceutical industry. The analysis of the microbiome could lead to better understanding of many chronic diseases, as well as unveil interesting compounds for a variety of treatments including new antibiotics. Finally, strategies such as TPD open the door to innovative therapies for neurodegenerative diseases and have attracted investment from industry. Other picks from past editions include rapid medical diagnostic tests and messenger RNA vaccines, two technologies that have become crucial during the COVID-19 pandemic.

IUPAC is especially keen on the impact of digital technologies in chemical discovery, thus another important pillar of the Top Ten initiative is identifying tools for digitalization. The world is adopting revolutionary digital tools such as artificial intelligence, robotics, big data and supercomputing - and so are chemists in academia and industry ${ }^{4}$. These technologies will transform our profession and have already yielded significant breakthroughs and created previously untapped opportunities ${ }^{5}$. Chemists of the future who are freed from more mundane and repetitive tasks by such technologies will have more time to dedicate to creative tasks as well as effective knowledge sharing ${ }^{6}$. Another example of IUPAC's pledge on digitalization is the creation of a chemical nomenclature for machines - the International Chemical Identifier (InChI; https://iupac.org/ who-we-are/divisions/division-details/ inchi/), which has become a key tool to advance machine reading and learning in chemistry. Pioneered by both IUPAC and the National Institute of Standards and Technology (NIST) in the United States, InChI enables chemical substances to be identified with a simple string of text, which is an essential tool for the findable, accessible, interoperable and reusable (FAIR) exchange, management and storage of digital chemical information.

With the Top Ten initiative, IUPAC aims to inspire chemists worldwide, especially early-career scientists and chemistry educators. Beyond this, the lists should also become a reference point for chemists, funding agencies, policymakers and other interested parties such as journalists and fact-checkers. Each of the technologies gives us a glimpse of what chemistry can achieve and how creativity and commitment for a more sustainable future can yield the solutions we so urgently need. They are also a call for multidisciplinarity and a more holistic way to teach, carry out and apply chemistry. One of the main goals of IUPAC is to implement a systems-thinking approach to the education, research and practice of chemistry.

In future years, the aim is to build broader and more diverse lists owing to suggestions made by chemists from around the world. We need global solutions with the potential to transform low- and middle-income countries into self-sufficient manufacturers - to guarantee access to chemicals, pharmaceuticals and sustainable energy solutions. The call for new submissions is now open until 31 March 2022 (https://bit.ly/SubmitTopTen22). Amid one of the biggest crises of our lifetime, chemistry has once again emerged as the answer: messenger RNA, lipid nanoparticles and antivirals, to name just a few examples. And chemistry will undoubtedly play a central part in tackling bigger long-term problems.

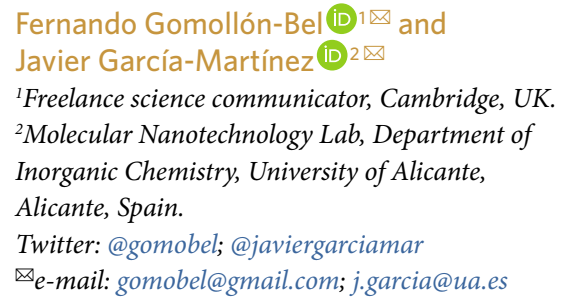

Published online: 31 January 2022 https://doi.org/10.1038/s41557-021-00887-9

References

1. Gomollón-Bel, F. Chem. Int. 41, 12-17 (2019).

2. García-Martínez, J. Angew. Chem. Int. Ed. 60, 4956-4960 (2021).

3. Ziegler, M. S. et al. Joule 3, 2134-2153 (2019).

4. García-Martínez, J. A vision for chemistry. Chem. Eng. News https://doi.org/10.1021/cen-09802-editorial (2020).

5. Baum, Z. J. et al. J. Chem. Inf. Model. 61, 3197-3212 (2021).

6. Milo, A. Isr. J. Chem. 58, 131-135 (2018).

Competing interests

F.G.-B. is paid by IUPAC to write the feature articles on the Top Ten Emerging Technologies in Chemistry. J.G.-M. is currently the president-elect of IUPAC and will be the president during 2022 and 2023 . 